\title{
Terahertz spectroscopy in biomedical field: a review on signal-to-noise ratio improvement
}

\author{
Yan Peng ${ }^{\dagger}$, Chenjun Shi ${ }^{\dagger}$, Yiming Zhu ${ }^{*}$, Min Gu and Songlin Zhuang
}

\author{
* Correspondence: ymzhu@usst.edu. \\ cn \\ ${ }^{\dagger}$ Yan Peng and Chenjun Shi are \\ contributed equally to this study \\ and share first authorship. \\ Terahertz Technology Innovation \\ Research Institute, Shanghai Key \\ Lab of Modern Optical System, \\ Terahertz Science Cooperative \\ Innovation Center, University of \\ Shanghai for Science and \\ Technology, Shanghai Institute of \\ Intelligent Science and Technology, \\ Shanghai, China
}

\begin{abstract}
With the non-ionizing, non-invasive, high penetration, high resolution and spectral fingerprinting features of terahertz $(\mathrm{THz})$ wave, $\mathrm{THz}$ spectroscopy has great potential for the qualitative and quantitative identification of key substances in biomedical field, such as the early diagnosis of cancer, the accurate boundary determination of pathological tissue and non-destructive detection of superficial tissue. However, biological samples usually contain various of substances (such as water, proteins, fat and fiber), resulting in the signal-to-noise ratio (SNR) for the absorption peaks of target substances are very small and then the target substances are hard to be identified. Here, we present recent works for the SNR improvement of THz signal. These works include the usage of attenuated total reflection (ATR) spectroscopy, the fabrication of sample-sensitive metamaterials, the utilization of different agents (including contrast agents, optical clearing agents and aptamers), the application of reconstruction algorithms and the optimization of $\mathrm{THz}$ spectroscopy system. These methods have been proven to be effective theoretically, but only few of them have been applied into actual usage. We also analyze the reasons and summarize the advantages and disadvantages of each method. At last, we present the prospective application of $\mathrm{THz}$ spectroscopy in biomedical field.
\end{abstract}

Keywords: Terahertz spectroscopy, SNR improvement, Metamaterial, ATR spectroscopy, Agent, Mixture algorithm, System optimization

\section{Introduction}

Terahertz $(\mathrm{THz})$ wave lies between the millimeter and infrared regions, whose frequency is from 0.1 to $10 \mathrm{THz}$ (corresponding to the wavelength from $0.3 \mathrm{~mm}$ to 30 $\mathrm{mm}$ ) [1]. Therefore, $\mathrm{THz}$ wave exhibits features of both sides. Some of the features, such as non-ionizing, non-invasive, high penetration, high resolution and spectral fingerprinting [2, 3], make $\mathrm{THz}$ wave a potential tool in biomedical field. Based on $\mathrm{THz}$ spectroscopy, many groups succeeded to obtain the fingerprint spectra of medicines [4-8], biomarkers [9-13], deoxyribonucleic acid (DNA) [14-17] and images of different cancers [18-25]. However, merely little work further studied the identification of biomarkers in actual biological samples, such as tissue, blood and urine. For these

(c) The Author(s). 2020 Open Access This article is licensed under a Creative Commons Attribution 4.0 International License, which permits use, sharing, adaptation, distribution and reproduction in any medium or format, as long as you give appropriate credit to the original author(s) and the source, provide a link to the Creative Commons licence, and indicate if changes were made. The images or other third party material in this article are included in the article's Creative Commons licence, unless indicated otherwise in a credit line to the material. If material is not included in the article's Creative Commons licence and your intended use is not permitted by statutory regulation or exceeds the permitted use, you will need to obtain permission directly from the copyright holder. To view a copy of this licence, visit http://creativecommons.org/licenses/by/4.0/. 
samples, various substances (such as water, proteins, fat, fiber and other organic components) are contained and the target substances often take a small part, resulting in the signal-to-noise ratio (SNR) for the absorption peaks of target substances are very small and then the target substances are hard to be identified.

Here, we present the related $\mathrm{THz}$ biomedical researches during the past few years working on the SNR improvement. We classify these works into three parts, as shown in Fig. 1.

The first part of SNR improvement for THz signal is about the biomedical sample treatment. Three methods are mainly discussed: attenuated total reflection (ATR) spectroscopy, metamaterial and agents (including contrast agents, optical clearing agents and aptamers). (1) ATR spectroscopy method is based on the interaction between sample and evanescent wave generated from crystal-sample interface, which is sensitive and needs few sample. However, as there is a wavenumber-dependent variation in penetration depth, the obtained ATR signal must be calibrated [26]. Furthermore, the evanescent wave interacts with each substance in the mixture sample, which makes it a total enhancement instead of specific/biomarker enhancement. (2) Metamaterial is a highly sensitive biosensor which can enhance SNR at specific frequency. There are two types of metamaterials: resonant frequency fixed metamaterials and resonant frequency tunable metamaterials. The frequency fixed metamaterial exhibits slight frequency shift of its resonant peaks when the sample covers on it (due to the change of total dielectric constant), therefore it can only reflect the change of dielectric constant, which cannot be used for the qualitative identification of target substances or biomarkers. The

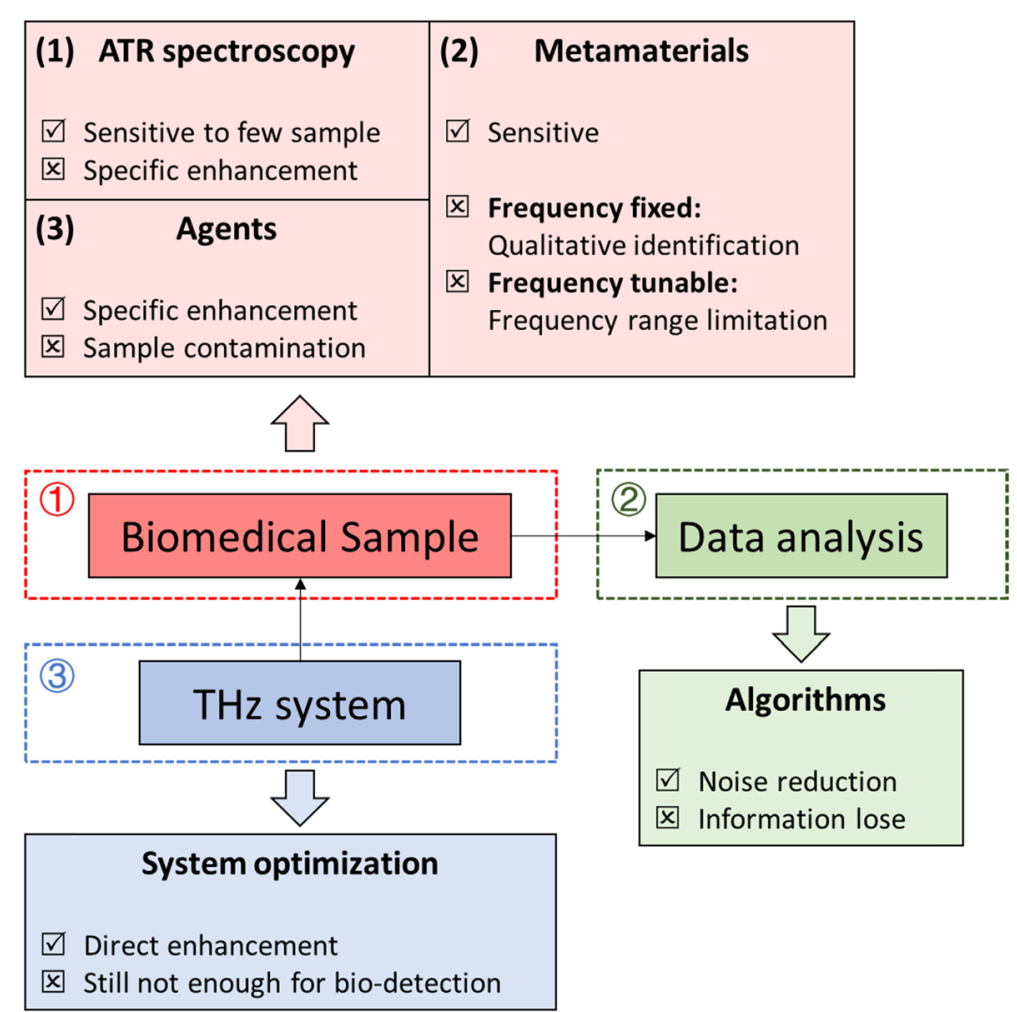

Fig. 1 Different methods to improve the SNR of THz signal according to the different parts of $\mathrm{THz}$ detection 
frequency tunable metamaterial can be used to obtain dielectric constant of sample at a wide range of frequency, which is essential for qualitative identification, but its frequency range is limited to the material's Fermi level. (3) Contrast agents use nanoparticles to enhance the contrast of adjacent areas during the $\mathrm{THz}$ imaging. Optical clearing agents are the liquids that transparent to $\mathrm{THz}$ wave. By washing the sample with these agents, water in the sample can be replaced by the agents, which can improve the transparent of sample for $\mathrm{THz}$ wave. Aptamers can bind with specific molecules such as amino acids, proteins or medicines, which can be used as a filter. However, the use of agent will cause sample contamination, which cannot be used for other tests.

The second part of SNR improvement for THz signal is the application of algorithms to reconstruct the data. This method can eliminate the irrelevant information and remain useful information of sample data, but during the data processing, some useful/ relevant information may be lost. Therefore, the use of algorithm needs to be optimized according to different samples or parameters.

For most biomedical studies based on $\mathrm{THz}$ spectroscopy, these two parts above are two main research directions for the SNR improvement without the modification of THz system. However, to detect these biomedical sample with high SNR, the most direct way is to enhance the intensity of $\mathrm{THz}$ source or the sensitivity of $\mathrm{THz}$ detector. Currently, many groups are specially study the optimization of the THz system. In the third part, we simply introduced the optimization works of the $\mathrm{THz}$ system that may be used in biomedical detection.

At the last of the paper, we present the prospective of $\mathrm{THz}$ spectroscopy applied in biomedical field. This review may provide some references for the further studies of signal enhancement methods.

\section{Treatment of biomedical sample}

The biomedical sample usually contains various substances, resulting in the SNR for the absorption peaks of target substances are very small and then the target substances are hard to be identified. Therefore, some treatments of biomedical samples are needed to enhance the THz resonance of target substances. Currently, using ATR spectroscopy, fabricating metamaterials and utilizing agents are three main research directions.

Different from the conventional THz spectroscopy, for THz ATR spectroscopy, a crystal is used to hold the sample and terahertz wave irradiates the crystal-sample interface to generate evanescent wave. The evanescent wave extends into and interacts with the sample [27], leading to the high sensitivity detection for the few sample. Therefore, many groups conducted biomedical researches based on ATR spectroscopy. In 2013, Shiraga et al presented a method to determine the complex dielectric constant of a cell monolayer based on ATR spectroscopy, whose imaginary part of the dielectric constant showed a lower absorption of slow relaxation mode than that of the liquid medium [28]. In 2015, Grognot et al measured permeabilization of epithelial cells based on ATR [29]. The cell layers displayed a $6 \%$ to $8 \%$ peak-amplitude relative contrast compared to the medium alone. In 2018, Zou et al monitored oxidative stress response of living human cells based on ATR THz time-domain spectroscopy [30]. As shown in Fig. 2, the dielectric constants were unchanged after the cell exposure to $\mathrm{H}_{2} \mathrm{O}_{2}$, while a slight increase of dielectric loss during time course of $\mathrm{H}_{2} \mathrm{O}_{2}$ exposing was observed. In 2019, 

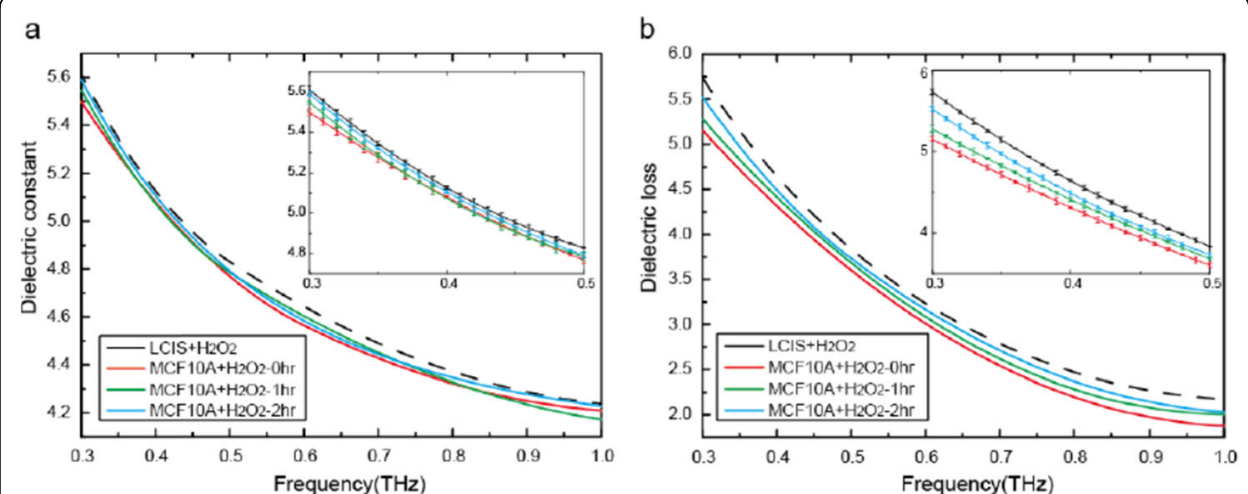

Fig. 2 a Dielectric constant and $\mathbf{b}$ dielectric loss of living cell after exposure to $10 \mathrm{mM} \mathrm{H}_{2} \mathrm{O}_{2}$ at 0 (red), $1 \mathrm{~h}$ (green) and $2 \mathrm{~h}$ (blue) were compared to the LCIS without cells added the same concentration of $\mathrm{H}_{2} \mathrm{O}_{2}$ (black). The inset shows the close-up of complex dielectric constants between 0.3 and $0.5 \mathrm{THz}$ [27]

Huang et al investigated the hydration state of amino acids based on $\mathrm{THz}$ time-domain ATR [31]. The results showed the decreasing of the dielectric loss with increasing in the L-threonine concentration within the band of $0.2 \sim 1.5 \mathrm{THz}$, and the lowest concentration they tested was $0.34 \mathrm{~mol} / \mathrm{L}$. Wang et al studied the dielectric characteristics of living glial-like cells by the ATR spectroscopy [32]. The experimental results showed that the dielectric responses were related significantly to the cell number, intracellular fluid, and cell structure, and they also found that the absorption of glioma cells was higher than that of normal cells.

Compared to the normal $\mathrm{THz}$ spectroscopy, the THz ATR spectroscopy shows a higher sensitivity. However, its sensitivity enhancement is for all the substances in the sample, which still cannot solve the problem of accurate recognition of the target biomarker.

Metamaterials are structure devices with a special designed layer usually made from metal or graphene, which is sensitive to the change of dielectric constant of sample. Therefore, metamaterial can reflect the subtle change of the sample itself. In past few years, many groups developed various kinds of metamaterials, whose enhanced frequency is fixed. In 2016, Hu et al constructed a metal microstructure array-dielectricmetal structure integrated with microfluidic. The sensitivity of their structure is 3.5 THz shifting per Refractive Units Index(THz/RIU) [33]. In 2017, Geng et al integrated two kinds of $\mathrm{THz}$ metamaterials biosensor with microfluidics as shown in Fig. 3. The two-gap-metamaterial was used to detect AFP and GGT-II (two kinds of liver cancer biomarker). The detection limit were about $19 \mathrm{GHz}$ resonance shift $(5 \mathrm{mu} / \mathrm{ml})$ and $14.2 \mathrm{GHz}$ resonance shift $(0.02524 \mu \mathrm{g} / \mathrm{ml})$ for GGT-II and AFP [34]. Al-Naib et al designed conductively coupled split ring resonators with the average sensitivity level of $3.0 \times 10^{4} \mathrm{~nm} / \mathrm{RIU} /$ unit-volume and up to $5.7 \times 10^{5} \mathrm{~nm} / \mathrm{RIU} /$ unit-volume at selected spots [35]. In 2018, Zhang et al designed a metamaterial biosensor with the sensitivity of $82 \mathrm{GHz} / \mathrm{RIU}$. Figure 4 shows the results of different amount of cancer cells HSC3 on the biosensor, which indicated the limit of the biosensor was approximately estimated to $1 \times 10^{5} \mathrm{cell} / \mathrm{ml}$ [36]. Qin et al demonstrated a metamaterial composed of metal ohm ring arrays, which is applied in detecting different concentrations of carbendazim. Resonant peaks of metamaterial move to a lower frequency as the concentration increases and the detection limit is $5 \mathrm{mg} / \mathrm{L}$, which is about 104 times enhancement compared to 


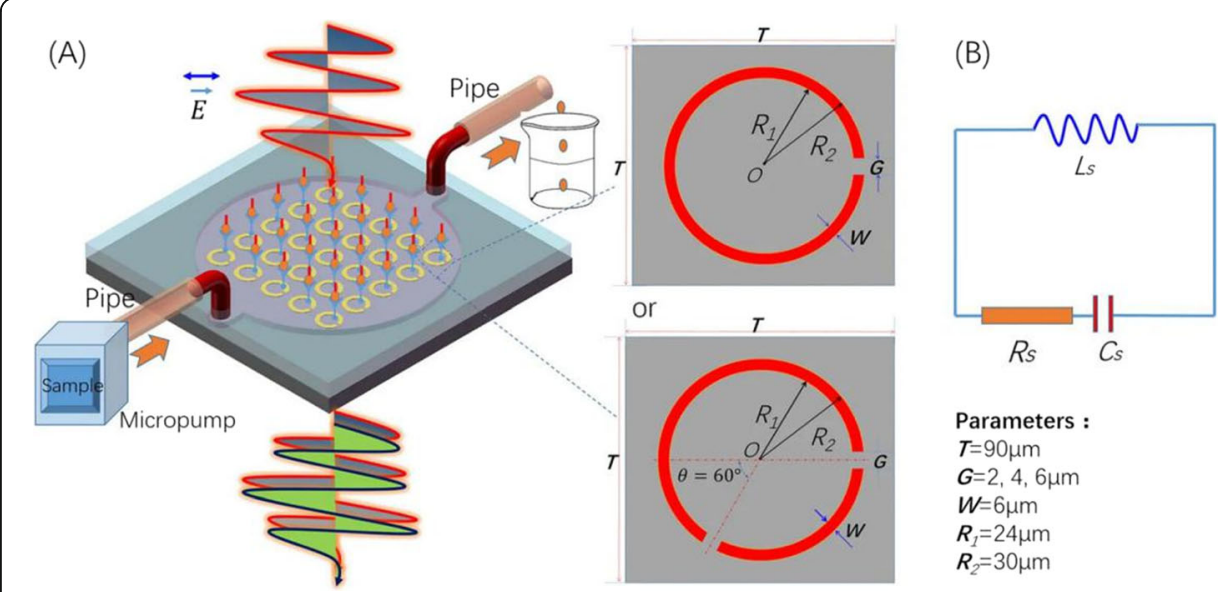

Fig. 3 a The sketch of THz metamaterials biosensor chip integrated with microfluidics; $\mathbf{b}$ Equivalent circuit with RLSs for the SRRs [34]

the squash method for THz-TDS detection [37]. Shin et al demonstrated a sensitive metamaterial with a metal array to detect 4-methylimidazole (4-MeI), a carcinogenic substance. The resonance frequency decreased and the transmittance increased as the 4-MeI concentration increased, shown in Fig. 5. The lowest concentration they tested is $1 \mathrm{mg} / \mathrm{L}$ [38]. Hong et al developed hybrid slot antenna structures with silver nanowires (AgNWs) were employed. The sensitivity increased upon the introduction of AgNWs with an enhancement factor of more than four times. They tested the devices using PRD1 viruses, and obtained an enhancement factor of 2.5 for a slot antenna width of $3 \mu \mathrm{m}$ [39]. 2019, Keshavarz et al demonstrated a metamaterial surface composed of an $\mathrm{H}$-shaped graphene resonator located on a semiconductor film. Three subtypes of Avian Influenza (AI) viruses, namely, H1N1, H5N2, and H9N2 viruses, were tested on the surface. The resonant frequency for H1N1, H5N2 and H9N2 were 1.668 $\mathrm{THz}, 1.665 \mathrm{THz}$ and1.641 THz, and the magnitude of the reflection were $61.4 \%, 67 \%$ and $60.9 \%$, respectively [40]. Zhao et al proposed $\mathrm{THz}$ metamaterial-based reflection spectroscopy for label-free sensing of living cells by a self-referenced method. The
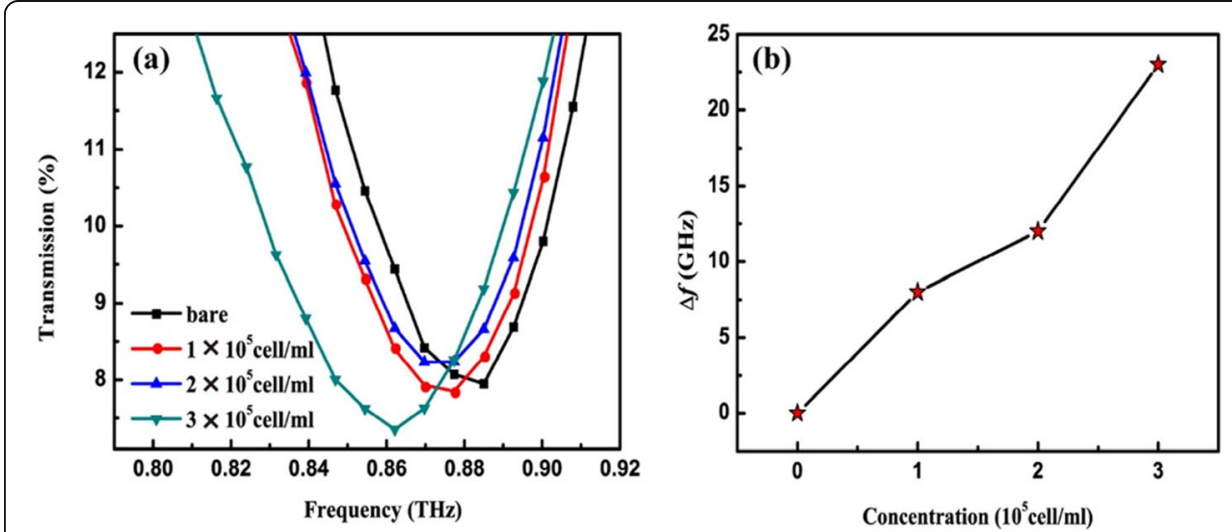

Fig. 4 a The measured transmission of the designed MMs-based biosensors under different cell concentrations, $1 \times 10^{5} \mathrm{cell} / \mathrm{ml}, 2 \times 10^{5} \mathrm{cell} / \mathrm{ml}$ and $3 \times 10^{5} \mathrm{cell} / \mathrm{ml}$, respectively. $\mathbf{b}$ The dependence of resonant frequency shift $\Delta f$ on cell concentration extracted from (a) [36] 


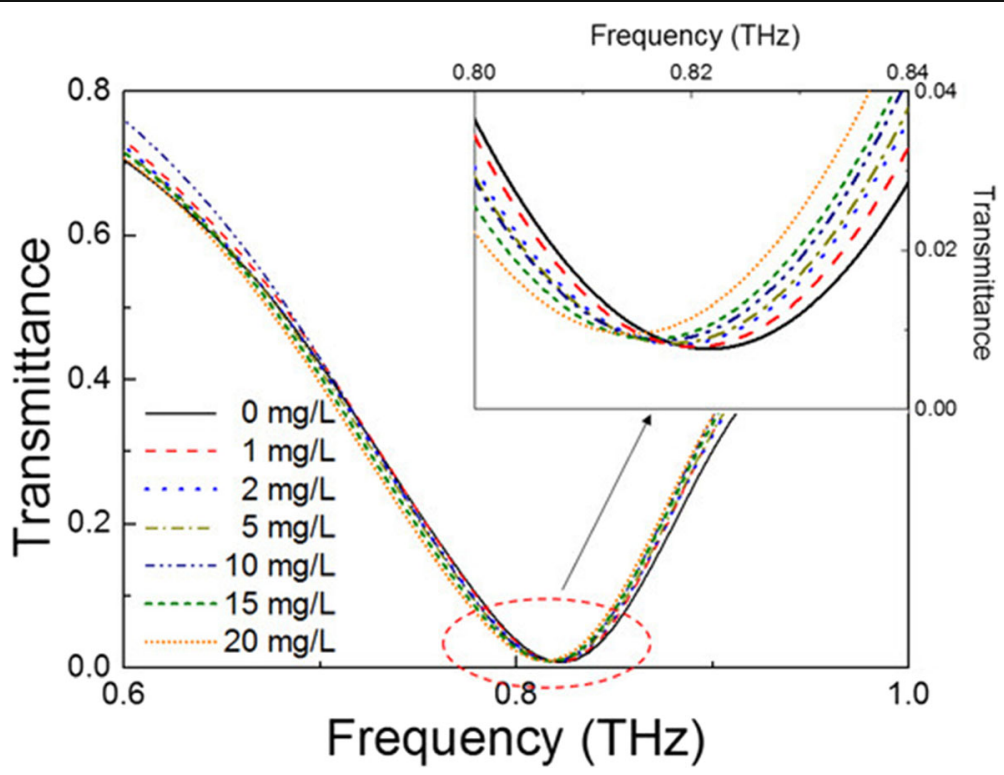

Fig. 5 Spectra of the 4-Mel detection, using the THz metamaterial, at concentrations of $0,1,2,5,10,15$, and $20 \mathrm{mg} / \mathrm{L}$ in the $\mathrm{THz}$ region of 0.6 to $1.0 \mathrm{THz}$ [38]

resonant peak intensity increase with increasing cell number, demonstrating a marked linearity with a linear correlation coefficient $\left(\mathrm{R}^{2}\right)$ of 0.9914 [41]. Roh et al investigated $\mathrm{f}$ two different $\mathrm{THz}$ metamaterials, namely double split ring resonator (DSRR) and the nano slot resonator (NSR), for molecule sensing in low concentration. Glucose and galactose were tested by the metamaterials with the detection limit of $0.1 \mu \mathrm{g} / \mu \mathrm{L}$ [42].

These metamaterials are proved to be sensitive sensors for biomedical detection. However, these studies only analyzed the resonance peaks of the metamaterials instead of biomedical sample, which means it cannot qualitatively identify the components in the biomedical sample. Therefore, recent researches are only limited to the single sample (only contains target substance, like standard medicine or single type of cell). This indicates that this method can only reflect the total dielectric constant (sample and metamaterial). Any changes of other substances will also cause the dielectric constant change of sample, which will change the total dialect constant and cause error in identification.

To realize the qualitative identification, the enhanced frequency of the metamaterial must be tunable to neutralize the influence of frequency shift caused by total dielectric constant change after sample covers on the metamaterial. These metamaterials can be made from graphene [43]. For example, in 2018, Tang et al designed a biosensor consisting of two sets of graphene micro-ribbon with different widths shown in Fig. 6, whose resonance peaks can be changed between $1.5 \mathrm{THz}$ to $4.5 \mathrm{THz}$ according to the change of the bias voltages [44]. Through the test of benzoic acid, their result showed the detection limit smaller than $6.35 \mu \mathrm{g} / \mathrm{cm}^{2}$. In 2019, Xu et al proposed a graphenemetamaterial heterostructure platform which can detect trace amount of chlorpyrifos methyl down to $0.2 \mathrm{ng}$ [45]. In 2020, Lee et al reported a graphene-combined nanoslot-based terahertz $(\mathrm{THz})$ resonance metamaterial to detect single-stranded deoxyribonucleic acids (ssDNAs) [46]. A maximum relative $\mathrm{THz}$ transmittance change of 52\% was observed with different types of ssDNA, with the detection limit of nmon $/ \mathrm{mm}^{2}$. 


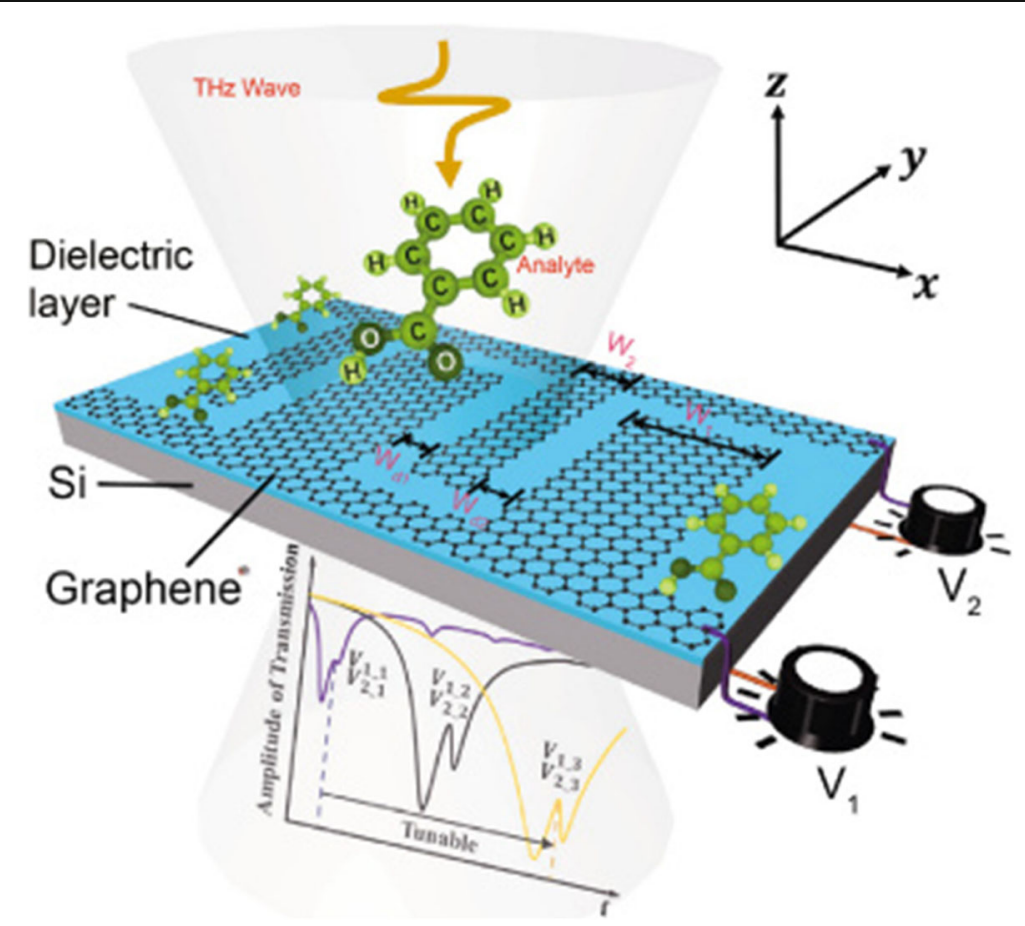

Fig. 6 Conceptual view of the PIT-sensor based on graphene micro-ribbon (GMR). The electromagnetic field is mainly concentrated at the edges of GMR, when excited by a THz wave, leading to the enhanced interaction between $\mathrm{THz}$ wave and the analyte. The tunability of the PIT sensor is achieved by changing the bias voltages (V1 and V2) applied on the two sets of GMR arrays [41]

These works of graphene metamaterials can adjust their resonance frequency to neutralize the frequency shift caused by total dielectric constant change after sample covers on the metamaterial, and then obtain samples' dielectric constant in a wide frequency range, which can be used for the qualitative and quantitative recognition of sample components. However, this frequency range is limited to the Fermi level of the metamaterial.

On the other side, some works utilized different kinds of agents to improve the SNR of $\mathrm{THz}$ detection. There are mainly three kinds of agents used in these studies: contrast agents, optical clearing agents and aptamers. These agents can enhance the imaging contrast, reduce water effects, or filter the target molecules. For example, in 2016, Zhang et al proposed the superparamagnetic iron oxide nanoparticles (SPIOs), which yields a highly sensitive increment in the reflection terahertz $(\mathrm{THz})$ upon exposure to an alternating magnetic field. They conducted focal-plane imaging experiments using water with and without $4 \mathrm{~g} / \mathrm{L}$ SPIOs, and the average amplitude in the relative reflection change images of water with SPIOs was $29.41 \% \pm 0.42 \%$, while that of water without SPIOs was only $0.30 \% \pm 0.03 \%$ [47]. In 2018, Musina et al used $\mathrm{THz}$ wave penetration-enhancing agents (PEA), including polyethylene glycol with different molecular weight, propylene glycol, ethylene glycol, and dimethyl sulfoxide, for optical clearing of tissues. The absorption of PEAs was about three times reduced compared to that of water [48]. In 2019, Hassan et al developed synthetic single stranded (ss) DNA aptamers as agents to be bind to mammaglobin B and mammaglobin A (two proteins overexpressed by breast cancer cells). As shown in Fig. 7, the agent can capture the 
cancer cell and neglect normal cells, resulting $\mathrm{THz}$ wave only having linear resonance with the concentration of cancer cells [49]. Huang et al designed silica-coated gold nanorods (GNRs) as a contrast agent for imaging of prostate cancer cells. As shown in Fig. 8, The enhancement efficiency of silica-coated GNRs was 5\% higher than that of uncoated GNRs and 25.35\% higher than that of the sample without nanoparticles [50]. Yang et al used fluorinated oil as an optical clearing agent to replace liquid medium around the living cell. By conducting an independent $\mathrm{t}$ test, statistically significant $\mathrm{THz}$ spectral differences were observed between the fluorinated oil with and without cells $(P<0.05$ at $0.5,1.0$, and $1.5 \mathrm{THz})$ [51]. Sadrara et al demonstrated hollow InSb microspheres forming dimers and trimers. Electric and magnetic hotspots in the gap between microspheres can be obtained, where electric field intensity enhancements of 10-2880 and magnetic field intensity enhancements of 3-61 in the frequency window $0.35-1.50$ $\mathrm{THz}[52]$.

These works of agent utilization successfully enhanced the characteristic absorption of specific substances, while the sample containment caused by the addition of extra substance is unavoidable. For some studies in vivo, the chosen agents must be no harmful to human body.

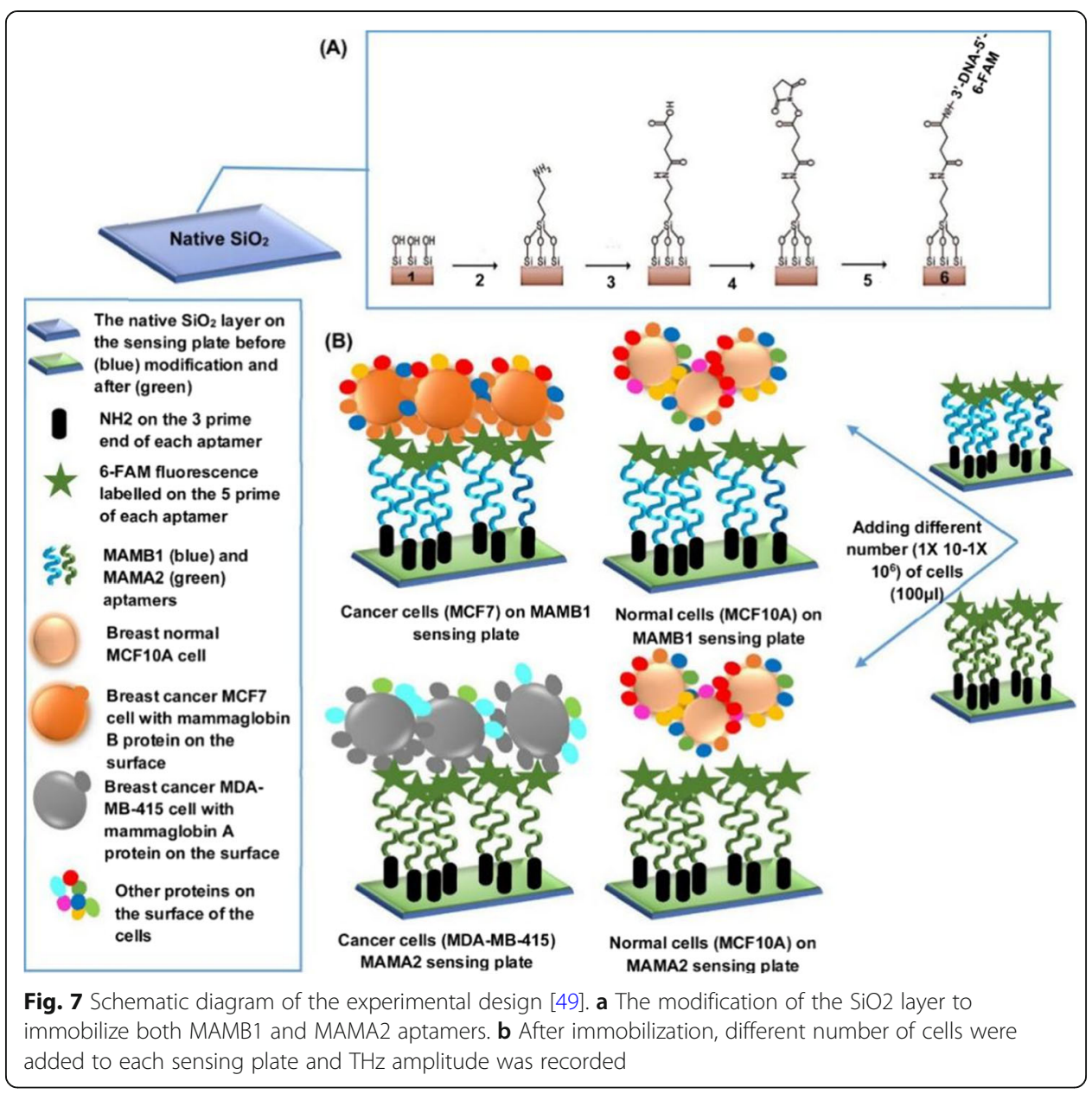




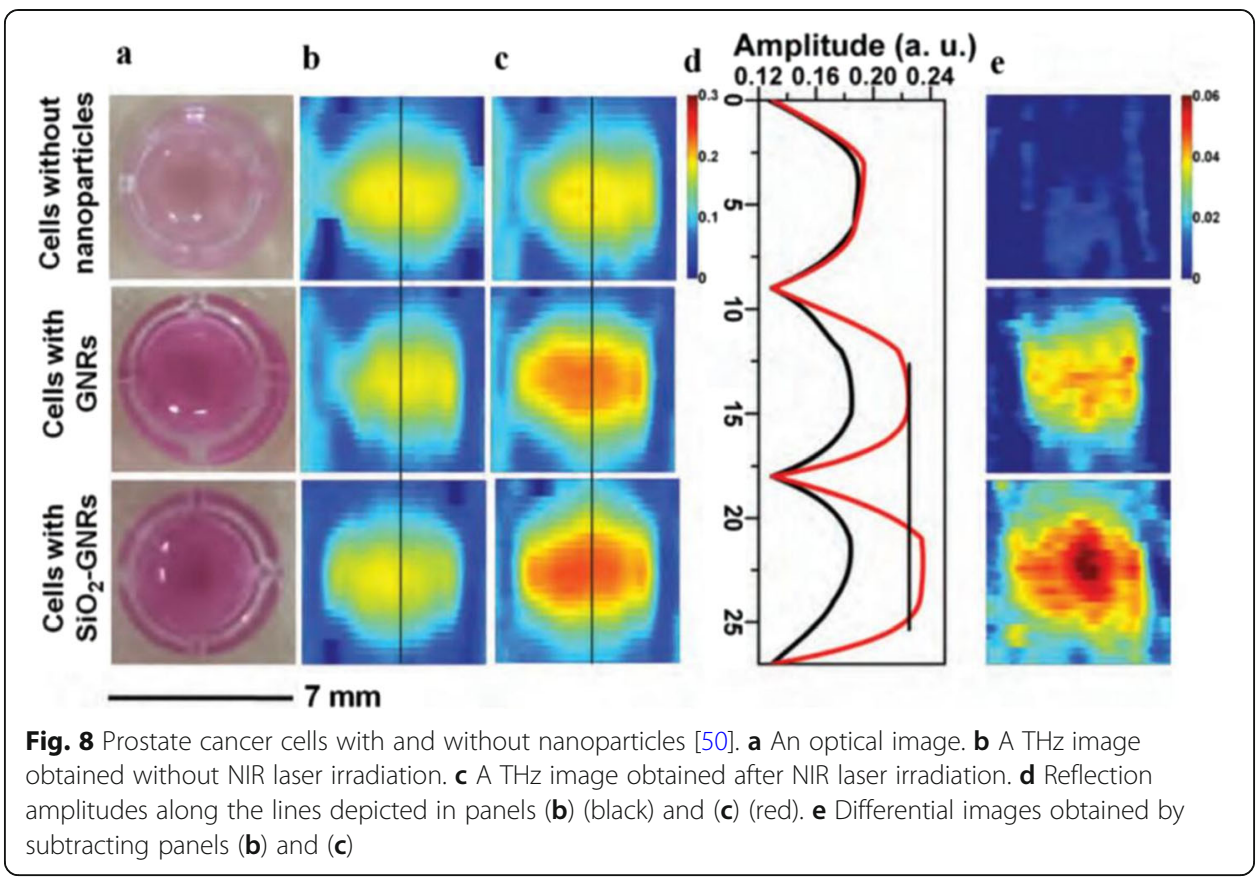

\section{Algorithms for data analysis}

After the detection of samples, various algorithms can be used to further improve the SNR of spectra. Currently, many studies use algorithms to denoise or reconstruct the data. This is an effective way to extract useful information from the spectra. These algorithms are used in the qualitative and quantitative identification of mixture samples, which can increase the identification accuracy.

For example, in 2016, Sterczewski used the Bayesian spectral source separation algorithm to estimate the dehydration kinetics of monohydrated D-glucose with high correlation coefficients of the linear fits to the dehydration model $(>0.90)$ were obtained [53]. From 2016 to 2017, Li et al proposed a self-adaptive genetic algorithm to decrease quantitative errors $[54,55]$. The quantitative analysis errors of 12 mixture samples were predominantly below 6\% with a standard deviation of 0.0344. In 2017, Qiao et al proposed the Mean Estimation Empirical Mode Decomposition (ME-EMD) de-noising algorithm. The algorithm flowchart is shown in Fig. 9. The de-noising results are shown in Table 1, which is better than that from "Db7" and "Sym8" [56]. Zou et al reported using principle component analysis (PCA) to reconstruct the time-domain $\mathrm{THz}$ signal, which can be used for the diagnosis of myelin deficit brain [57]. According to the results of mice samples, the first two PCs explain the $85 \%$ of time-domain information and basing on the two PCs, myelin deficit brain and normal brain can be classified with the accuracy of $96.7 \%$. In 2018, Petrov et al presented the iterative and self-healing algorithm for imaging resolution enhancement, 1.21 times enhancement of reconstruction quality in low noise and 1.34 times enhancement in higher noise conditions can be obtained by the algorithm [58]. Peng et al proposed qualitative analysis algorithm based on the wavelet transform, baseline elimination, support vector regression, and loop iteration of samples. The spectra before and after denoising process are shown in Fig. 10. The average correlation coefficient of identification reached $99.135 \%$ and the rootmean-square error reached $0.40 \%$ [59]. Liu et al demonstrated a self-adaptive algorithm 


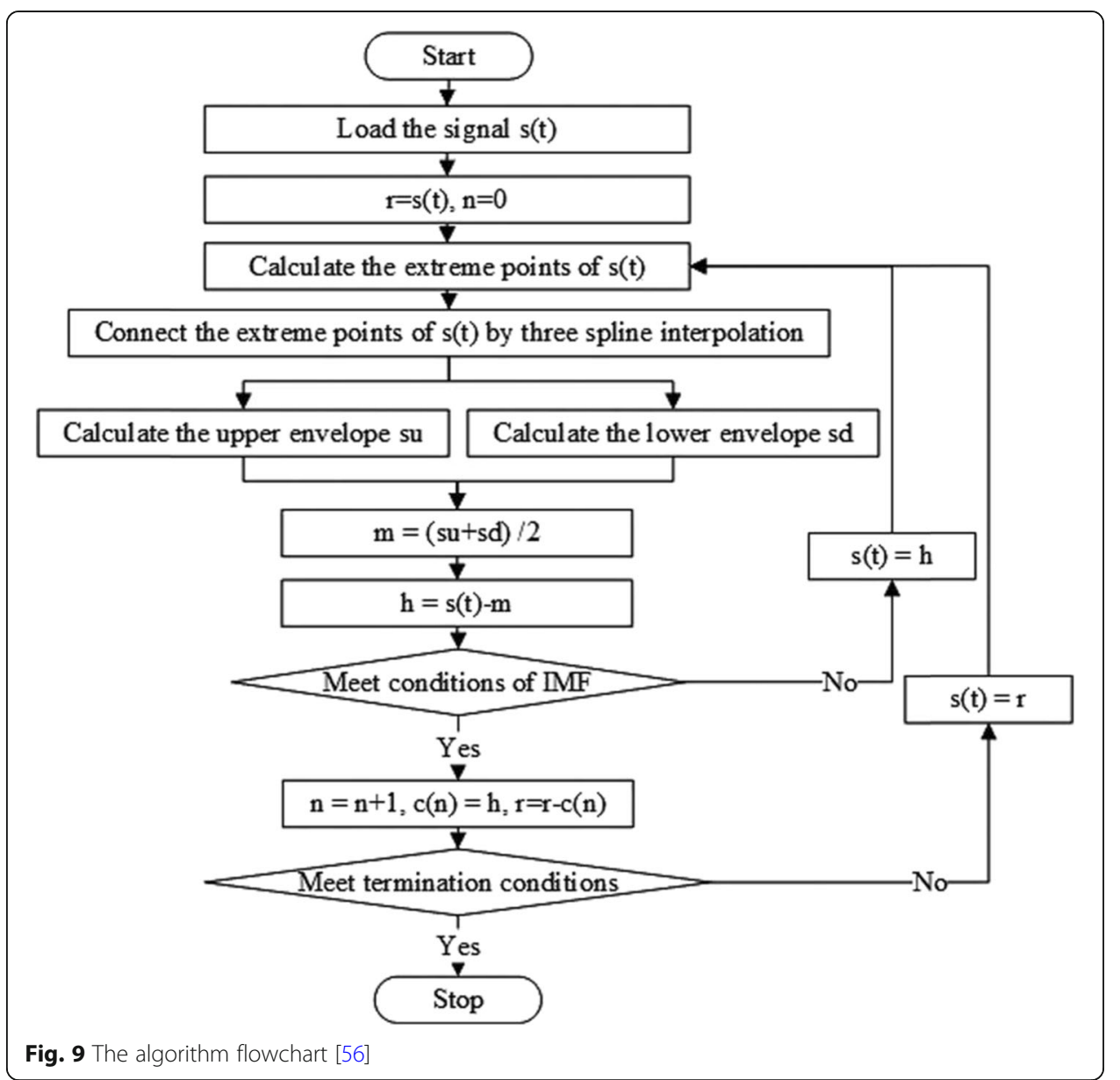

based on the Hilbert-Huang transform or identifying and eliminating atmospheric vapor noise from $\mathrm{THz}$ spectra. Monolayer graphene (MG) was tested, showing that strong interference of high-humidity atmospheric water vapor was eliminated and the characteristic peak of MG near $0.7 \mathrm{THz}$ was retained [60]. In 2019, Cui et al applied lifting wavelet transform based on different wavelet basis function to the denoising of terahertz time domain spectrum, achieving a SNR of $60.69 \mathrm{~dB}$ and a least RMSE of $2.85 \times 10^{-5}$ [61]. Huang et al composited multiscale entropy (CMSE) method and clustered by the $\mathrm{K}$-means algorithm to extract $\mathrm{THz}$ features of glycoproteins. The average accuracy of their methods was $84.46 \%$, while results from the method of PCA only reached $72.22 \%[62]$.

Algorithms can process the signal data to extract effective information and eliminate irrelative information. Therefore, these algorithms can also improve the SNR of spectra indirectly and then realize the effective identification of target substances. In particular, algorithms normally have various parameters that should be properly set according to

Table 1 Results of the Simulation Experiment [56]

\begin{tabular}{lllll}
\hline Materials & Original & Db7 & Sym8 & ME-EMD \\
\hline SNR & 21.7557 & 24.5401 & 24.5587 & 25.3761 \\
MSE & 0.0033 & 0.0024 & 0.0024 & 0.0022 \\
\hline
\end{tabular}



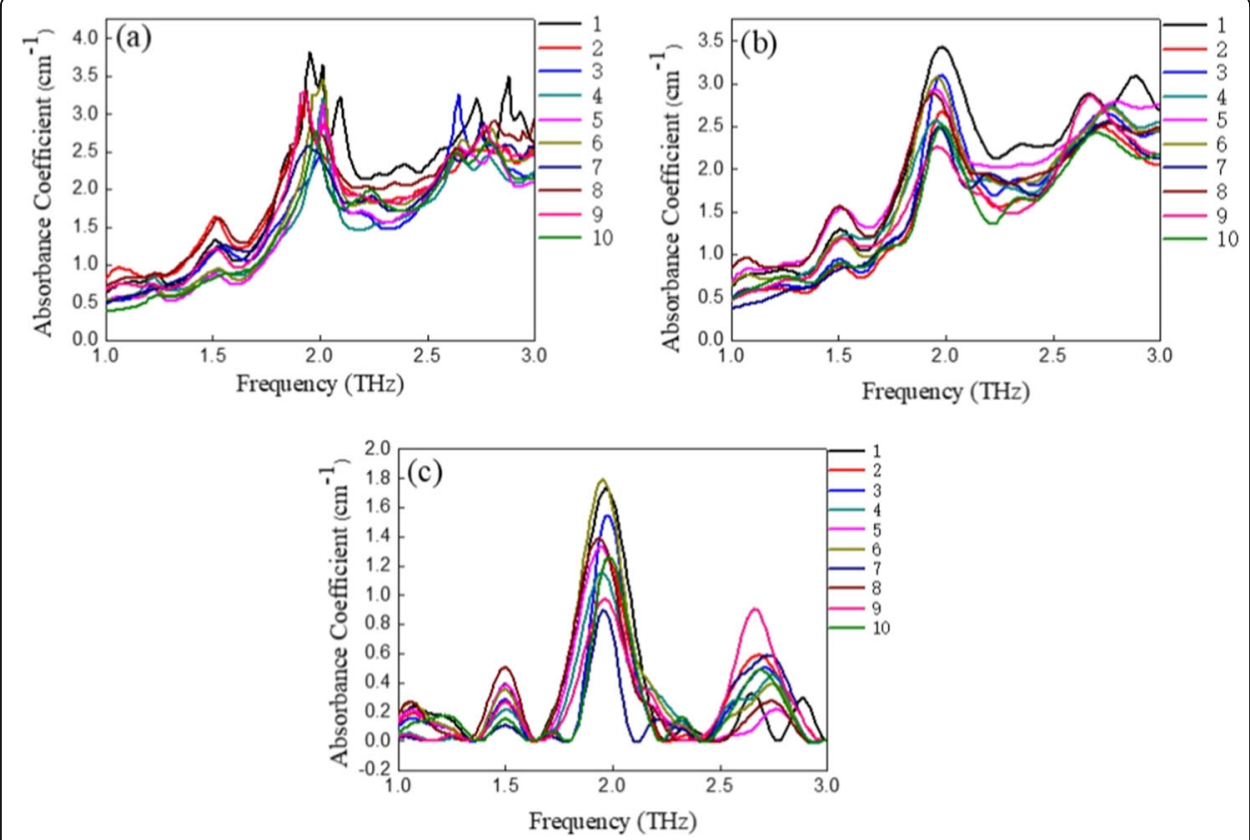

Fig. $10 \mathrm{THz}$ absorption spectra of ten mixture samples [59]. a Original spectra. b Spectra after wavelet transform. c Baseline correction by polynomial fitting

the data. Therefore, wrong setting of the parameters may lead to the information loss of the sample and cause errors in the final spectral recognition.

\section{Optimization of THz system}

To detect the THz signal passing through the biomedical sample with higher SNR, the most direct way is to enhance the intensity of $\mathrm{THz}$ source or the sensitivity of $\mathrm{THz}$ detectors. Here, we also simply introduced current works of THz systems' optimization.

$\mathrm{THz}$ wave can be generated by many methods including photoconductive antennas, optical rectification, electron accelerator, and laser filament, where photoconductive antennas can generate $\mathrm{THz}$ pulses signals without the need of high-power optical sources [63] and became a classical $\mathrm{THz}$ resource for $\mathrm{THz}$ spectroscopy. However, the biomedical sample usually contains water and other substances which can strongly absorb $\mathrm{THz}$ wave. The antenna can't provide enough $\mathrm{THz}$ energy for high SNR detection. Therefore, many works focus on the improvement of antenna to obtain the stronger THz signal.

For example, in 2016, Zarrabi et al presented an antenna with cross-shaped nanoaperture structure. By Finite-difference time-domain method analyzing, the field intensity for the antenna with the structure was enhanced by 28.2 times. After added graphene coat and a cross shape chain of silicon dioxide to the structure, the intensity was enhanced by 43.9 times [64]. Zangeneh-Nejad et al proposed a hybrid graphene molybdenum disulphide-based photoconductive antenna, which provides not only high input impedance and reconfigurability but also high values of matching efficiency and radiation efficiency. The simulated results showed the total efficiency of the antenna increases by several orders of magnitude [65]. Collier et al introduced $\mathrm{THz}$ antennas 
utilizing textured InP semiconductors which shortened carrier lifetimes - ultimately reducing Joule heating and ohmic losses. The carrier lifetimes of the smooth-, fine-, and coarse-textured InP semiconductors are found as respective values of $200 \pm 6,100 \pm 10$, and $20 \pm 3$ ps when measured with a pump-probe experimental system [66]. In 2017, Amanatiadis et al showed a graphene plasmonic antenna via synthesized substrates with metamaterial resonators, whose radiation efficiency is calculated at $16.6 \%$, more than four times higher compared to the 3.9\% of the usual antenna [67]. In 2018, Gupta et al reported several factors of enhancement in $\mathrm{THz}$ emission efficiency from conventional antennas. By coating an $80 \mathrm{~nm}$ nano-layer of dielectric $\left(\mathrm{TiO}_{2}\right)$ on the active area between the electrodes of a semi-insulating GaAs-based device, shown in Fig. 11, the reflection from the Si-GaAs interface was suppressed by 6.9\% [68]. In 2019, Kazemi et al used mixed graphene-gold electrodes as electrode material of the antenna, which enhanced the detected $\mathrm{THz}$ signal peak amplitude up to 14.31\% [69]. Korolev et al reported using nanoimprint lithography to enhance $\mathrm{THz}$ emission from $\mathrm{MAPbI}_{3}$ perovskite upon femtosecond laser irradiation. The efficiency of $\mathrm{THz}$ emission from the nanostructured perovskite was enhanced by 3.5 times as compared with a smooth perovskite film [70]. In 2020, Cheng et al coated $\mathrm{THz}$ antenna by an epsilon-near-zero (ENZ) metamaterial superstrate showed in Fig. 12, enhanced the peak gain of the antenna by $45 \%$ from $5.37 \mathrm{~dB}$ to $7.79 \mathrm{~dB}$ [71].

Additionally, there are also some studies about other kinds of $\mathrm{THz}$ sources, including two-color laser excitation, spintronic $\mathrm{THz}$ emitters, photomixers, and filamentation. For example, in 2018, Jin et al used intense two-color laser pulses exciting liquid water to generate $\mathrm{THz}$ wave. Compared with one-color excitation, Their method enhanced the THz energy by two-orders of magnitude [72]. In 2019, Chen et al demonstrated spintronic $\mathrm{THz}$ emitters combined with semiconductor materials. A 2-3 order enhancement of the $\mathrm{THz}$ signals in a lower $\mathrm{THz}$ frequency range $(0.1-0.5 \mathrm{THz})$ is observed [73]. Ironside et al projected a metamaterial-enhanced photomixer shown in Fig. 13(b) compared to the conventional photomixer shown in Fig. 13(a) which generate $\mathrm{THz}$ powers in the milliwatt range and exceed the Manley-Rowe limit for frequencies less than $2 \mathrm{THz}$ [74]. In 2020, Koulouklidis et al demonstrated two-color filamentation of femtosecond mid-infrared laser pulses at $3.9 \mu \mathrm{m}$ generating ultrashort

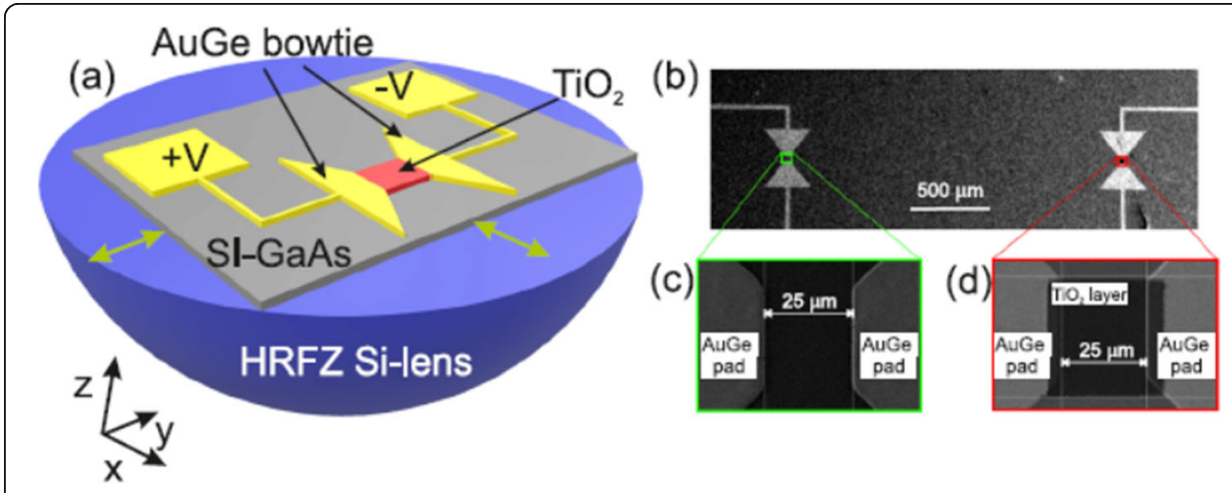

Fig. 11 a Schematic view of an antenna on SI-GaAs with $\mathrm{TiO}_{2}$ layer coating. The green arrows indicate the micrometer controlled alignment of the substrate on the HRFZ Si-lens. $\mathbf{b}$ SEM image of the uncoated and $\mathrm{TiO}_{2}$-coated antenna on the same substrate. $\mathbf{c}$ and $\mathbf{d}$ show the zoomed-in images of the regions marked by the green and the red squares in (b), respectively (90 rotated) [68] 
sub-cycle $\mathrm{THz}$ pulses with sub-milijoule energy. The results showed $\mathrm{THz}$ conversion efficiency of 2.36\%, resulting in THz field amplitudes above $100 \mathrm{MV} \mathrm{cm}^{-1}$ [75].

Also, some studies focus on the sensitivity improvement of the detector basing on antenna optimization by additional structure or layers. If the detector can be more sensitive, then the weak signal after passing through biological sample can be effectively detected. In 2016, Mou et al demonstrated a THz detector chip consisting of a differentially fed antenna and a Schottky diode at its terminal, as shown in Fig. 14. The chip showed the gain enhancement of 5.4-7.7 dB within the operating bandwidth [76]. In 2017, Xiao et al designed a grating-coupled structure on the high-resistivity silicon substrate to enhance the ability of coupling terahertz signals. The electric field in the central area of the silicon surface can be enhanced more than 4 times compared with the non-structure silicon substrate [77]. In 2018, Siday et al demonstrated an efficient terahertz $(\mathrm{THz})$ detector based on an optical hybrid cavity. By putting an optically thin photoconductive layer between a distributed Bragg reflector and an array of electrically isolated nanoantennas, the sensitivity is enhanced by $17 \%$ [78]. In 2019, Cheng et al proposed sandwiching hyperbolic metamaterials composed of $\mathrm{InSb}$ and $\mathrm{SiO} 2$ multilayer with hole arrays. The layer was put between a terahertz dipole antenna and the substrate, which enhanced the near-field electric field intensities by more than three times [79].

Besides the devices optimization, the external factors control is another useful option to increase the SNR of THz signal, such as reduce the humidity of environment, avoid shocks during the measurement and maintain the stability of temperature. Specifically, the improvement of this way may enhance the signal of all substances in the sample, which makes the signal of target substances still be drowned out by that of other substances. Therefore, this method can be an assistant to the previous two methods (sample treatments and algorithms) for the further SNR improvement.

\section{Discussion}

These works improve the SNR of biomedical signal from different aspects such as sample treatment, reconstruction algorithms for data analysis and system optimization. For the treatment of sample, there are three directions. Some works measured the sample

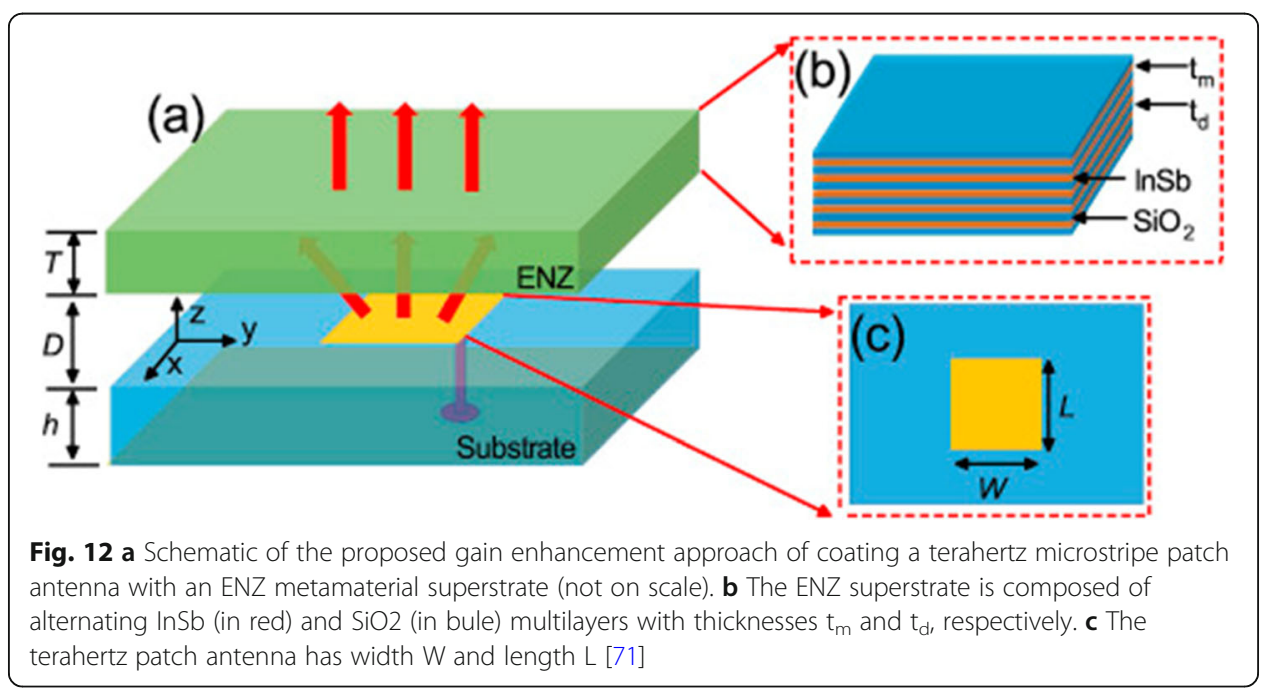




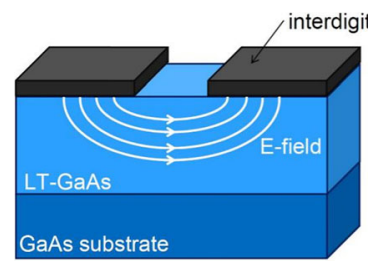

(a) conventional

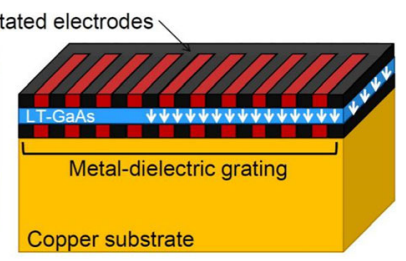

(b) metamaterial

Fig. 13 As illustration depicting key photomixer design features between (a) the conventional photomixer design and (b) the proposed enhanced metamaterial design [74]

using THz ATR system. This is an effective way to improve SNR but it enhances the resonance of all components in the sample. When the concentration of target biomarker is small, it will still be difficult to identify the biomarker in the mixture sample. Some works tested metamaterials with sample covered. For the metamaterials that their resonant frequency is fixed, they can only analyze the shifting of the resonance peaks to achieve quantitative analysis, which can't be used for qualitative identification of the sample. For the metamaterials that their resonant frequency is tunable, they can adjust the resonant frequency to get the dielectric constant of sample in a wide range of frequency for qualitative and quantitative analysis, but this frequency range is limited to the Fermi level of the metamaterial. Also, change of other substances in the sample will cause error in the final result. On the other hand, some works utilized different agents to enhance the imaging contrast, reduce water effects or filter target molecules. These works realized the resonance enhancement of specific substances, but it will cause sample containment. For the improvement of data analysis methods, many algorithms were established. These algorithms can extract the feature information of target substances in the sample and reduce the effect of noise, which also improve the final SNR. Many works realized the qualitative and quantitative analysis of mixture biomedical sample with higher accuracy based on these algorithms. Most biomedical studies based on $\mathrm{THz}$ spectroscopy improved the signal SNR by these three directions, which did not need to modify the THz system. However, the optimization of system is the most direct way to improve the SNR. Currently, some groups specially studied the optimization of $\mathrm{THz}$ system. We introduced the optimization works that may be used in biomedical detection. However, as signals of all substances in the sample are enhanced in this

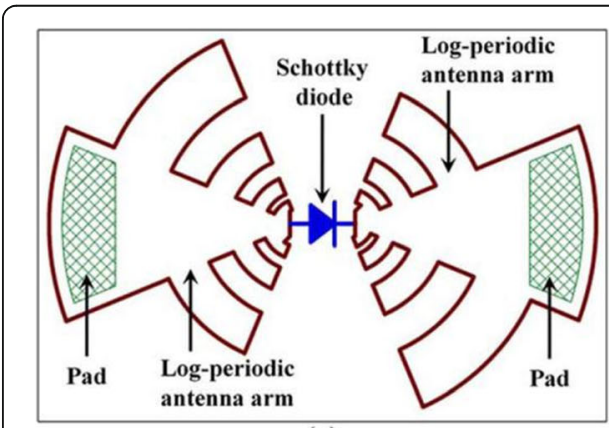

(a)

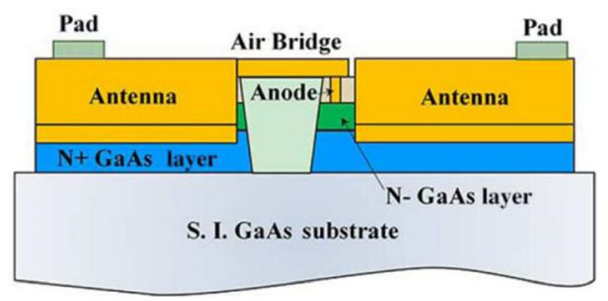

(b)

Fig. 14 Structure of the detectenna chip [76]. a Topology. b Vertical structure 
method, which makes the signal of target substance still be drown out by the signal of other substances.

We hope that through this review, readers can get broad understanding of current methods for signal enhancement of $\mathrm{THz}$ biomedical detection. For the researchers having difficulty in the $\mathrm{THz}$ detection of biomedical sample, this review can provide some experiences which may be helpful. Also, for the future researches on the signal enhancement, here we give our prospective. Sample treatment, data analysis and system optimization methods can be combined, which may further improve the SNR of biomedical sample. Furthermore, sample derivatization can be another direction to improve SNR. If the target substance shows low resonance to $\mathrm{THz}$ wave, we can find some chemical reaction that is specific to this substance and transfer the target substance to a new substance with a higher resonance. Also, other substances in the sample that show high resonance to $\mathrm{THz}$ wave can be eliminated by this way.

\section{Conclusions}

In this review, we introduced current works for SNR improvements of biomedical signal, including sample treatment (including applying ATR spectroscopy, fabricating metamaterials and adding agents), reconstruction algorithms and system optimization. Each has its advantages and disadvantages:

- ATR spectroscopy is sensitive to few sample but it cannot specifically enhance the resonance of target substances.

- Metamaterials are sensitive biosensors, in which resonant frequency fixed metamaterials can reflect the total change of dielectric constant, but can only be used for quantitative analysis. On the other hand, resonant frequency tunable metamaterials can be used to get the spectra of sample in a frequency range for qualitative and quantitative analysis. However, this frequency range is limited to the Fermi level of the metamaterial.

- Agents can be used for specific enhancement of substances but will cause sample containment.

- Algorithms can extract effective information from redundancy information but some useful information will be lost during the process.

- System optimization is the most direct way to enhance the $\mathrm{THz}$ signal, but enhancement of all signal makes the signal of target substance still be drown out by the signals of other substances.

Through these methods introduced, reader can get broad understanding of the approaches of signal improvement during biomedical detection based on $\mathrm{THz}$ spectroscopy. And at last in the discussion, we give our prospective of the future researches on the signal enhancement.

Abbreviations

THz: Terahertz; SNR: Signal-to-Noise Ratio; RUI: Refractive Units Index; ATR: Attenuated total reflection

Acknowledgements

Not applicable.

Authors' contributions

Y.P. and Y.M. Z. developed the idea and supervised the project. C.J. S. contributed to the research of current studies.

Y. P. and C.J. S. were major contributors in writing the manuscript. All authors revised the paper and approved the final manuscript. 


\section{Funding}

National Major Project of Scientific Instrument and Equipment Development (2017YFF0106300); National Natural Science Foundation of China (61922059, 61771314, 61722111, 81961138014); Shanghai Rising-Star Program (17QA1402500), the 111 Project (D18014), the International Joint Lab Program supported by Science and Technology Commission Shanghai Municipality (17590750300), the Key project supported by Science and Technology Commission Shanghai Municipality (YDZX20193100004960).

\section{Availability of data and materials}

The datasets used and/or analysed during the current study are available from the corresponding author on reasonable request.

\section{Competing interests}

The authors declare that they have no competing interests.

Received: 24 January 2020 Accepted: 11 March 2020

\section{Published online: 13 April 2020}

\section{References}

1. Pickwell E, Wallace V. Biomedical applications of terahertz technology. J Phys D Appl Phys. 2006;39(17):R301.

2. Danciu M, Alexa-Stratulat T, Stefanescu C, Dodi G, Tamba BI, Mihai CT, Stanciu GD, Luca A, Spiridon IA, Ungureanu LB. Terahertz spectroscopy and imaging: a cutting-edge method for diagnosing digestive cancers. Materials. 2019;12(9): 1519.

3. Peng Y, Zhu Y, Gu M, Zhuang S. Terahertz spatial sampling with subwavelength accuracy. Light Sci Appl. $2019 ; 8: 72$.

4. Zeitler JA, Kogermann K, Rantanen J, Rades T, Taday PF, Pepper M, Aaltonen J, Strachan CJ. Drug hydrate systems and dehydration processes studied by terahertz pulsed spectroscopy. Int J Pharm. 2007;334(1-2):78-84.

5. Kawase K, Ogawa Y, Watanabe $Y$, Inoue H. Non-destructive terahertz imaging of illicit drugs using spectral fingerprints. Opt Express. 2003;11(20):2549-54.

6. Sibik J, Löbmann K, Rades T, Zeitler JA. Predicting crystallization of amorphous drugs with terahertz spectroscopy. Mo Pharm. 2015;12(8):3062-8

7. Davies AG, Burnett AD, Fan W, Linfield EH, Cunningham JE. Terahertz spectroscopy of explosives and drugs. Mater Today. 2008;11(3):18-26.

8. Taday PF, Bradley I, Arnone D, Pepper M. Using terahertz pulse spectroscopy to study the crystalline structure of a drug: a case study of the polymorphs of ranitidine hydrochloride. J Pharm Sci. 2003;92(4):831-8.

9. Li T, Ma H, Peng Y, Chen X, Zhu Z, Wu X, Kou T, Song B, Guo S, Liu L. Gaussian numerical analysis and terahertz spectroscopic measurement of homocysteine. Biomed Optics Express. 2018;9(11):5467-76.

10. Chen W, Peng Y, Jiang X, Zhao J, Zhao H, Zhu Y. Isomers identification of 2-hydroxyglutarate acid disodium salt (2HG) by terahertz time-domain spectroscopy. Sci Rep. 2017;7(1):12166.

11. Altan H, Ozek NS, Gok S, Ozyurt I, Severcan F. Monitoring of tryptophan as a biomarker for cancerous cells in Terahertz (THz) sensing. Paper present in Optical Biopsy XIV: Toward Real-Time Spectroscopic Imaging and Diagnosis, SPIE BiOS, San Francisco, California, United States, 2016

12. Joseph CS, Yaroslavsky AN, Al-Arashi M, Goyette TM, Dickinson JC, Gatesman AJ, Soper BW, Forgione CM, Horgan TM, Ehasz EJ. Terahertz spectroscopy of intrinsic biomarkers for non-melanoma skin cancer. In: Terahertz Technology and Applications II, SPIE OPTO: Integrated Optoelectronic Devices, San Jose, California, United States; 2009.

13. Wu X, Dai Y, Wang L, Peng Y, Lu L, Zhu Y, Shi Y, Zhuang S. Diagnosis of methylglyoxal in blood by using far-infrared spectroscopy and o-phenylenediamine derivation. Biomedical Optics Express. 2020;11(2):963-70.

14. Fischer B, Walther M, Jepsen PU. Far-infrared vibrational modes of DNA components studied by terahertz time-domain spectroscopy. Phys Med Biol. 2002;47(21):3807.

15. Brucherseifer $M$, Nagel $M$, Haring Bolivar $P$, Kurz H, Bosserhoff $A$, Büttner R. Label-free probing of the binding state of DNA by time-domain terahertz sensing. Appl Phys Lett. 2000;77(24):4049-51.

16. Markelz A, Roitberg A, Heilweil EJ. Pulsed terahertz spectroscopy of DNA, bovine serum albumin and collagen between 0.1 and 2.0 THz. Chem Phys Lett. 2000;320(1-2):42-8.

17. Cheon H, Yang H-j, Lee S-H, Kim YA, Son J-H. Terahertz molecular resonance of cancer DNA. Sci Rep. 2016;6:37103.

18. Oh SJ, Kang J, Maeng I, Suh J-S, Huh Y-M, Haam S, Son J-H. Nanoparticle-enabled terahertz imaging for cancer diagnosis. Opt Express. 2009;17(5):3469-75.

19. Brun M-A, Formanek F, Yasuda A, Sekine M, Ando N, Eishii Y. Terahertz imaging applied to cancer diagnosis. Phys Med Biol. 2010;55(16):4615.

20. Nakajima S, Hoshina H, Yamashita M, Otani C, Miyoshi N. Terahertz imaging diagnostics of cancer tissues with a chemometrics technique. Appl Phys Lett. 2007;90(4):041102.

21. Woodward RM, Cole BE, Wallace VP, Pye RJ, Arnone DD, Linfield EH, Pepper M. Terahertz pulse imaging in reflection geometry of human skin cancer and skin tissue. Phys Med Biol. 2002;47(21):3853.

22. Fitzgerald AJ, Wallace VP, Jimenez-Linan M, Bobrow L, Pye RJ, Purushotham AD, Arnone DD. Terahertz pulsed imaging of human breast tumors. Radiology. 2006;239(2):533-40.

23. Woodward R, Wallace V, Arnone D, Linfield E, Pepper M. Terahertz pulsed imaging of skin cancer in the time and frequency domain. J Biol Phys. 2003;29(2-3):257-9.

24. Ashworth PC, Pickwell-MacPherson E, Provenzano E, Pinder SE, Purushotham AD, Pepper M, Wallace VP. Terahertz pulsed spectroscopy of freshly excised human breast cancer. Opt Express. 2009;17(15):12444-54.

25. Meng K, T-n C, Chen T, Zhu L-g, Liu Q, Li Z, Li F, S-c Z, Z-r L, Feng H. Terahertz pulsed spectroscopy of paraffinembedded brain glioma. J Biomed Opt. 2014;19(7):077001

26. Newnham DA, Taday PF. Pulsed terahertz attenuated total reflection spectroscopy. Appl Spectrosc. 2008;62(4):394-8.

27. Shen Y-C. Terahertz pulsed spectroscopy and imaging for pharmaceutical applications: a review. Int J Pharm. 2011; $417(1-2): 48-60$. 
28. Shiraga K, Ogawa Y, Suzuki T, Kondo N, Irisawa A, Imamura M. Determination of the complex dielectric constant of an epithelial cell monolayer in the terahertz region. Appl Phys Lett. 2013;102(5):053702.

29. Grognot M, Gallot G. Quantitative measurement of permeabilization of living cells by terahertz attenuated total reflection. Appl Phys Lett. 2015;107(10):103702.

30. Zou Y, Liu Q, Yang X, Huang H-C, Li J, Du L-H, Li Z-R, Zhao J-H, Zhu L-G. Label-free monitoring of cell death induced by oxidative stress in living human cells using terahertz ATR spectroscopy. Biomed Optics Express. 2018;9(1):14-24

31. Huang H, Liu Q, Zhu L, Zou Y, Li Z, Li Z. Dual-prism based terahertz time-domain attenuated total reflection spectroscopy and its application to characterise the hydration state of L-threonine in solution. Opt Commun. 2019;437: $133-8$.

32. Wang Y, Jiang Z, Xu D, Chen B, Wang S, Mu N, Chen T, Feng H, Yao J. Dielectric responses of living glial cell monolayer based on terahertz ATR spectroscopy. In: Infrared, Millimeter-Wave, and Terahertz Technologies VI, SPIE/COS Photonics Asia, Hangzhou, China, 2019.

33. Hu X, Xu G, Wen L, Wang H, Zhao Y, Zhang Y, Cumming DR, Chen Q. Metamaterial absorber integrated microfluidic terahertz sensors. Laser Photonics Rev. 2016;10(6):962-9.

34. Geng Z, Zhang X, Fan Z, Lv X, Chen H. A route to terahertz metamaterial biosensor integrated with microfluidics for liver cancer biomarker testing in early stage. Sci Rep. 2017;7(1):16378.

35. Al-Naib I. Biomedical sensing with conductively coupled terahertz metamaterial resonators. IEEE J Selected Topics Quantum Electronics. 2016;23(4):1-5.

36. Zhang Z, Ding $H$, Yan $X$, Liang L, Wei D, Wang M, Yang Q, Yao J. Sensitive detection of cancer cell apoptosis based on the non-bianisotropic metamaterials biosensors in terahertz frequency. Optical Materials Express. 2018;8(3):659-67.

37. Qin B, Li Z, Hu F, Hu C, Chen T, Zhang H, Zhao Y. Highly sensitive detection of carbendazim by using terahertz timedomain spectroscopy combined with metamaterial. IEEE Transact Terahertz Sci Technol. 2018;8(2):149-54.

38. Shin H, Jang H, Ok G. Highly sensitive detection of 4-Methylimidazole using a terahertz Metamaterial. Sensors. 2018; 18(12):4304.

39. Hong J, Jun S, Cha S, Park J, Lee S, Shin G, Ahn Y. Enhanced sensitivity in THz plasmonic sensors with silver nanowires. Sci Rep. 2018;8(1):15536.

40. Keshavarz A, Vafapour Z. Sensing avian influenza viruses using terahertz metamaterial reflector. IEEE Sensors J. 2019; 19(13):5161-6.

41. Zhao X, Lin Z, Wang Y, Yang X, Yang K, Zhang Y, Peng J, de la Chapelle ML, Zhang L, Fu W. Label-free self-referenced sensing of living cells by terahertz metamaterial-based reflection spectroscopy. Biomed Optics Express. 2019;10(3):1196206.

42. Roh Y, Lee S-H, Kang B, Wu JW, Ju B-K, Seo M. Terahertz optical characteristics of two types of metamaterials for molecule sensing. Opt Express. 2019;27(13):19042-9.

43. Rodrigo D, Limaj O, Janner D, Etezadi D, De Abajo FJG, Pruneri V, Altug H. Mid-infrared plasmonic biosensing with graphene. Science. 2015;349(6244):165-8.

44. P-r T, Li J, L-h D, Liu Q, Q-x P, J-h Z, Zhu B, Z-r L, Zhu L-g. Ultrasensitive specific terahertz sensor based on tunable plasmon induced transparency of a graphene micro-ribbon array structure. Opt Express. 2018;26(23):30655-66

45. Xu W, Xie L, Zhu J, Tang L, Singh R, Wang C, Ma Y, Chen H-T, Ying Y. Terahertz biosensing with a graphenemetamaterial heterostructure platform. Carbon. 2019;141:247-52.

46. Lee S-H, Choe J-H, Kim C, Bae S, Kim J-S, Park Q-H, Seo M. Graphene assisted terahertz Metamaterials for sensitive biosensing. Sensors Actuators B Chem. 2020;310:127841.

47. Zhang R, Zhang L, Wu T, Zuo S, Wang R, Zhang C, Zhang J, Fang J. Contrast-enhanced continuous-terahertz-wave imaging based on superparamagnetic iron oxide nanoparticles for biomedical applications. Opt Express. 2016;24(8): 7915-21.

48. Musina GR, Dolganova IN, Malakhov KM, Gavdush AA, Chernomyrdin NV, Tuchina DK, Komandin GA, Chuchupal SV, Cherkasova OP, Zaytsev KI. Terahertz spectroscopy of immersion optical clearing agents: DMSO, PG, EG, PEG. Paper presented at Millimetre Wave and Terahertz Sensors and Technology XI, SPIE Security + Defence, 2018, Berlin, Germany.

49. Hassan EM, Mohamed A, DeRosa MC, Willmore WG, Hanaoka Y, Kiwa T, Ozaki T. High-sensitivity detection of metastatic breast cancer cells via terahertz chemical microscopy using aptamers. Sensors Actuators B Chem. 2019;287:595-601.

50. Huang Q, Zou Y, Zhong S, Yang X, Li J, Huang W, Zhu H, Cheng C, Ding M, Zhu L-G. Silica-coated gold Nanorods with high Photothermal efficiency and biocompatibility as a contrast agent for in vitro terahertz imaging. J Biomed Nanotechnol. 2019;15(5):910-20

51. Yang K, Yang X, Zhao X, Lamy de la Chapelle M, Fu W. THz spectroscopy for a rapid and label-free cell viability assay in a microfluidic Chip based on an optical clearing agent. Anal Chem. 2018;91(1):785-91.

52. Sadrara M, Miri M. Electric and magnetic hotspots via hollow Insb microspheres for enhanced terahertz spectroscopy. Sci Rep. 2019;9(1):2926.

53. Sterczewski LA, Grzelczak MP, Nowak K, Szlachetko B, Plinski E. Bayesian separation algorithm of THz spectral sources applied to D-glucose monohydrate dehydration kinetics. Chem Phys Lett. 2016;644:45-50.

54. Li Z. Wavelength selection for quantitative analysis in terahertz spectroscopy using a genetic algorithm. IEEE Transact Terahertz Sci Technol. 2016;6(5):658-63.

55. Li Z, Guan A, Ge H, Lian F. Wavelength selection of amino acid THz absorption spectra for quantitative analysis by a self-adaptive genetic algorithm and comparison with mwPLS. Microchem J. 2017;132:185-9.

56. Qiao X, Zhang X, Ren J, Zhang D, Cao G, Li L. Mean estimation empirical mode decomposition method for terahertz time-domain spectroscopy de-noising. Appl Opt. 2017;56(25):7138-45.

57. Zou Y, Li J, Cui Y, Tang P, Du L, Chen T, Meng K, Liu Q, Feng H, Zhao J. Terahertz spectroscopic diagnosis of myelin deficit brain in mice and rhesus monkey with chemometric techniques. Sci Rep. 2017;7(1):1-9.

58. Petrov NV, Balbekin NS, Kulya MS, Gorodetsky AA. Reconstruction enhancement of noisy data in terahertz pulse timedomain holography by iterative procedure. Paper presented at Unconventional Optical Imaging, SPIE Photonics Europe, Strasbourg, France, 2018.

59. Peng Y, Shi C, Xu M, Kou T, Wu X, Song B, Ma H, Guo S, Liu L, Zhu Y. Qualitative and quantitative identification of components in mixture by terahertz spectroscopy. IEEE Transact Terahertz Sci Technol. 2018;8(6):696-701. 
60. Liu H, Fan Y-X, Li L, Chen H-G, Wang P-F, Tao Z-Y. Self-adaptive terahertz spectroscopy from atmospheric vapor based on Hilbert-Huang transform. Opt Express. 2018;26(21):27279-93.

61. Cui G, Peng W, Liu Y, Chang CA. Denoising algorithm for terahertz time domain spectrum based on lifting wavelet transform. In: Second Symposium on Novel Technology of X-Ray Imaging, Second Symposium on Novel Technology of X-Ray Imaging, Hefei, China, 2018.

62. Huang P, Huang Z, Lu X, Cao Y, Yu J, Hou D, Zhang G. Study on glycoprotein terahertz time-domain spectroscopy based on composite multiscale entropy feature extraction method. Spectrochimica Acta Part A Mol Biomol Spectrosc. 2019;229:117948.

63. Lepeshov S, Gorodetsky A, Krasnok A, Rafailov E, Belov P. Enhancement of terahertz photoconductive antenna operation by optical nanoantennas. Laser Photonics Rev. 2017;11(1):1600199.

64. Zarrabi FB, Naser-Moghadasi M, Heydari S, Maleki M, Arezomand AS. Cross-slot nano-antenna with graphene coat for bio-sensing application. Opt Commun. 2016;371:34-9.

65. Zangeneh-Nejad F, Safian R. Significant enhancement in the efficiency of photoconductive antennas using a hybrid graphene molybdenum disulphide structure. J Nanophotonics. 2016;10(3):036005.

66. Collier CM, Krupa JD, Hristovski IR, Stirling TJ, Bergen MH, Holzman JF. Textured semiconductors for enhanced photoconductive terahertz emission. In: Terahertz, RF, Millimeter, and Submillimeter-Wave Technology and Applications IX, SPIE OPTO, San Francisco, California, United States, 2016.

67. Amanatiadis SA, Karamanos TD, Kantartzis NV. Radiation efficiency enhancement of graphene THz antennas utilizing metamaterial substrates. IEEE Antennas Wireless Propagation Lett. 2017;16:2054-7.

68. Gupta A, Rana G, Bhattacharya A, Singh A, Jain R, Bapat RD, Duttagupta S, Prabhu S. Enhanced optical-to-THz conversion efficiency of photoconductive antenna using dielectric nano-layer encapsulation. APL Photonics. 2018;3(5): 051706.

69. Kazemi AH, Mahani FF, Mokhtari A. Peak amplitude enhancement of photoconductive antenna using periodic nanoslit and graphene in the THz band. Optik. 2019;185:114-20.

70. Korolev VI, Pushkarev AP, Obraztsov PA, Tsypkin AN, Zakhidov AA, Makarov SV. Enhanced terahertz emission from imprinted halide perovskite nanostructures. Nanophotonics. 2019;9(1):187-94.

71. Cheng C, Lu Y, Zhang D, Ruan F, Li G. Gain enhancement of terahertz patch antennas by coating epsilon-near-zero metamaterials. Superlattices Microstructures. 2020;139:106390.

72. Jin Q, Dai J, EY, Zhang X-C. Terahertz wave emission from a liquid water film under the excitation of asymmetric optical fields. Appl Phys Lett. 2018;113(26):261101.

73. Chen M, Wu Y, Liu Y, Lee K, Qiu X, He P, Yu J, Yang H. Current-enhanced broadband THz emission from Spintronic devices. Adv Optical Mater. 2019;7(4):1801608.

74. Ironside DJ, Salas R, Chen P-Y, Le KQ, Alú A, Bank SR. Enhancing THz generation in photomixers using a metamaterial approach. Opt Express. 2019;27(7):9481-94.

75. Koulouklidis AD, Gollner C, Shumakova V, Fedorov VY, Pugžlys A, Baltuška A, Tzortzakis S. Observation of extremely efficient terahertz generation from mid-infrared two-color laser filaments. Nat Commun. 2020;11(1):1-8.

76. Mou J, Xue Q, Guo D, Lv X. A THz detector chip with printed circular cavity as package and enhancement of antenna gain. IEEE Trans Antennas Propag. 2016;64(4):1242-9.

77. Xiao P, Tu X, Jiang C, Wan C, Jiang Z, Zhai S, Gu M, Jia X, Kang L, Chen JA. A Sensitive coupling structure for terahertz detectors array. In: Terahertz, RF, Millimeter, and Submillimeter-Wave Technology and Applications X, SPIE OPTO, San Francisco, California, United States, 2017

78. Siday T, Thompson RJ, Glass S, Luk T-S, Reno JL, Brener I, Mitrofanov O. An efficient terahertz detector based on an optical hybrid cavity. Paper presented at Terahertz, RF, Millimeter, and Submillimeter-Wave Technology and Applications XI, SPIE OPTO, San Francisco, California, United States, 2018.

79. Cheng C, Chen W, Lu Y, Ruan F, Li G. Large near-field enhancement in terahertz antennas by using hyperbolic Metamaterials with hole arrays. Appl Sci. 2019;9(12):2524.

\section{Publisher's Note}

Springer Nature remains neutral with regard to jurisdictional claims in published maps and institutional affiliations.

\section{Submit your manuscript to a SpringerOpen ${ }^{\circ}$ journal and benefit from:}

- Convenient online submission

- Rigorous peer review

- Open access: articles freely available online

- High visibility within the field

- Retaining the copyright to your article

Submit your next manuscript at $\boldsymbol{\nabla}$ springeropen.com 